oesophagus, 4 of 7 (57.1\%) oesophageal squamous lesions and 6 of $39(15.3 \%)$ gastric lesions. All patients were discussed at a multidisciplinary meeting and those patients who were fit were offered radical surgery or chemoradiotherapy. Six patients who were offered radical surgery opted for conservative management with endoscopic follow-up. 14 patients proceeded to radical surgery; six of these had no residual cancer in surgical specimen and eight had residual cancer present. 11 of the 14 are currently in disease free survival, two died of recurrence and one died of post-operative complications. Two patients received radical chemoradiotherapy; one is in disease free survival, the other died of advanced adenocarcinoma. One patient received radical radiotherapy and remains free of recurrence. Nine patients received conservative/endoscopic management; of these seven had disease free survival, two died of metastatic adenocarcinoma. Mean follow-up was 32 months.

Conclusion Our results show that submucosal invasion is found in a significant proportion of patients undergoing upper gastrointestinal ER. Management of SM invasive cancer following ER remains challenging and our series shows a wide variation in management outcomes. Further research to guide the optimum management of this group of patients is required.

Competing interests None declared.

\section{PWE-025 POST-RADIOTHERAPY PHARYNGEAL/PROXIMAL OESOPHAGEAL STRICTURES IN HEAD AND NECK MALIGNANCY: OUTCOME OF ENDOSCOPIC BOUGIE DILATION}

doi:10.1136/gutjnl-2012-302514d.25

${ }^{1} \mathrm{R}$ Sringeri, ${ }^{2} \mathrm{~L}$ Priestnall, ${ }^{3} \mathrm{R}$ Cullen, ${ }^{1} \mathrm{~N}$ C Fisher. ${ }^{1}$ Department of Gastroenterology, Birmingham, UK; ${ }^{2}$ Speech and Language Therapy Department, Birmingham, UK; ${ }^{3} E N T$, The Dudley Group NHS Foundation Trust, Birmingham, UK

Introduction Chemo-radiotherapy is the standard of care for most patients with head and neck malignancy. Radiotherapy may lead to dysphagia due to pharyngeal/proximal oesophageal strictures. Endoscopic management of these strictures with antegrade dilation by using Savary Gilliard bougie dilators is described in literature. ${ }^{12}$ Our aim was to review the outcome in patients referred for bougie dilation for radiotherapy induced strictures.

Methods It is a retrospective case notes review.

Results 11 patients underwent bougie dilation of radiotherapy induced strictures in last 4 years. Nine male, two female patients with median age of 71 years. Five patients had laryngeal cancer and six had oropharyngeal cancer. All patients had radiotherapy where four had concurrent chemotherapy. Five patients required flouroscopy and seven patients required nase endoscope. Median size of initial bougie size was $11 \mathrm{~mm}$ and final bougie size was $17 \mathrm{~mm}$. Mean number of procedures per patient was 4 . No complications noted. While three patients had good response, six had borderline and two had none. Median interval from completion of radiotherapy to index procedure was 2.5 years with range from 2 months to 12 years.

Conclusion Savary Gilliard bougie dilation appears to be safe and well tolerated method for dilating pharyngeal/proximal oesophageal strictures secondary to radiotherapy treatment for head and neck cancer. While symptom improvement varied among patients probably early intervention might benefit the patient.

Competing interests None declared.

\section{REFERENCES}

1. Ahlawat SK, Al-Kawas FH. Endoscopic management of upper esophageal strictures after treatment of head and neck malignancy. Gastrointest Endosc 2008;68:19-24.

2. Dhir V, Vege SS, Mohandas KM, et al. Dilation of proximal esophageal strictures following therapy for head and neck cancer: experience with Savary Gilliard dilators. J Surg Oncol 1996;63:187-90.

\section{PWE-026 INFRARED SPECTROSCOPY ACURATELY DETECTS} BARRETT'S MUCOSA BIOPSY SPECIMENS EX VIVO

doi:10.1136/gutjnl-2012-302514d.26

${ }^{1,2} \mathrm{R}$ J Haidry, ${ }^{* 1,2}$ L B Lovat, ${ }^{2} \mathrm{M}$ Banks, ${ }^{1} \mathrm{~A}$ Gupta, ${ }^{1} \mathrm{M}$ A Butt, ${ }^{3} \mathrm{M}$ Novelli, ${ }^{4} \mathrm{~T}$ Kosciolek ${ }^{4}$ A Marechal, ${ }^{4}$ P Rich. ${ }^{1}$ Department of Surgery, National Medical Laser Centre, London, UK; ${ }^{2}$ Department of Gastroenterology, London, UK; ${ }^{3}$ Department of Histopathology, University College Hospital, London, UK; ${ }^{4}$ Institute of Structural and Molecular Biology, University Collge London, London, UK

Introduction Oesophageal cancer (OAC) arises in Barrettt's oesopahgus (BE) and carries a poor prognosis. Early mucosal neoplasia arising in $\mathrm{BE}$ can be treated successfully with minimally invasive endotherapy. Fourier transform infrared spectroscopy (FTIR) can detect specific molecules from their unique vibrational absorption spectra in complex materials like human tissue. There is growing literature on it's medical diagnostic uses in the mid-infrared (MID-IR) range of $1800-900 \mathrm{~cm}^{-1}$

Methods 98 biopsy specimens were obtained from 21 patients undergoing endoscopy for BE surveillance over 3 months. 30 were from squamous epithelium, at least $2 \mathrm{~cm}$ above the squamocolumnar junction \& remainder from visible BE mucosa. At each site a biopsy was taken for MID-IR analysis. A matched biopsy was taken from the same site for histopathological grading. FTIR spectra were recorded on biopsies at room temperature with a Bruker IFS 66/S spectrometer fitted with a liquid nitrogen-cooled MCT-B detector and an Attenuated Total Reflection silicon microprism. For each spectrum, 1000 interferograms (approximately 2 min accumulation time) were averaged before Fourier transformation. Spectra were converted to second derivatives to remove baseline artefacts and improve signal resolution. An automated programme was used to quantify specific characteristic features and normalise them relative to intensities of the protein amide II band in the same spectrum. The results were used to calculate their correlation with presence of glandular mucosa in BE.

Results Normal squamous mucosa and BE could be differentiated with a sensitivity of $82 \%$ and specificity of $96 \%$ by analysing variations in the $1180-1000 \mathrm{~cm}^{-1}$ region of second derivative of spectra. Bands in this region responsible for the observed differences arise from variations in levels of glycogen or a related material within the tissues. BE tissue appear to have at least 50\% lower concentrations compared to the squamous epithelium.

Conclusion FTIR spectroscopy can accurately differentiate between the columnar mucosa of $\mathrm{BE}$ and normal squamous oesophagus. Further work is underway to examine the accuracy of this technique in differentiating different states of dysplasia in BE. IR spectroscopy provides a fast and effective means of detecting $\mathrm{BE}$ ex vivo and presents an exciting avenue of future research with a view to incorporating IR spectral analysis into existing technologies to capture real time spectral data at endoscopy to help guide endotherapy to these high risk patients.

Competing interests None declared.

\section{PWE-027 HALO RADIOFREQUENCY ABLATION FOR SQUAMOUS HIGH GRADE DYSPLASIA AND EARLY SQUAMOUS CELL CARCINOMA: OUTCOMES FORM THE UK HALO RADIOFREQUENCY ABLATION REGISTRY}

doi:10.1136/gutjnl-2012-302514d.27

${ }^{1,2} \mathrm{R}$ J Haidry, ${ }^{*}{ }^{1} \mathrm{~J}$ Dunn, ${ }^{2} \mathrm{M}$ Banks, ${ }^{1} \mathrm{~A}$ Gupta, ${ }^{1} \mathrm{M}$ A Butt, ${ }^{3} \mathrm{H}$ Smart, ${ }^{4} \mathrm{P}$ Bhandari, ${ }^{5} \mathrm{~L}$ - A Smith, ${ }^{6} \mathrm{R}$ Willert, ${ }^{7} \mathrm{G}$ Fullarton, ${ }^{8} \mathrm{M}$ Di Pietro, ${ }^{9}$ I Penman, ${ }^{10} \mathrm{M}$ Novelli, ${ }^{1,2} \mathrm{~L}$ B Lovat. ${ }^{1}$ Department of Surgery, National Medical Laser Centre, London, UK; ${ }^{2}$ Department of Gastroenterology, University College Hospital, London, UK; ${ }^{3}$ Department of Gastroenterology, Royal Liverpool University Hospital, Liverpool, UK; ${ }^{4}$ Department of Gastroenterology, Princess Alexandra Hospital, Portsmouth, UK; ${ }^{5}$ Department of 